\title{
Computing Technology: Special Announcement COMPsych: The electronic software information service and PC software archive for psychology
}

\author{
PETER A. HORNBY and MARGARET D. ANDERSON \\ State University of New York College at Plattsburgh, Plattsburgh, New York
}

\begin{abstract}
COMPsych is an electronic clearinghouse for software information related to psychology instruction, research, and practice. Since 1987, COMPsych has provided five major services: (1) a catalog of descriptive information about available software, (2) a directory of software users, (3) a message system, (4) an announcement service, and (5) an electronic Newsletter. In addition to these services, COMPsych has now been designated as the official archive and distribution site for PC-based software and data files described in the journal Behavior Research Methods, Instruments, \& Computers. A general description of COMPsych and specific instructions for using the new archive service are presented. Those interested in joining COMPsych should send electronic mail to compsych@snyplava.bitnet or compsych@splava.cc.plattsburgh.edu. The system can be accessed via anonymous ftp at gluon.hawk.plattsburgh.edu in the directory pub/compsych. COMPsych files are also available through the gopher server at SUNY Plattsburgh.
\end{abstract}

COMPsych was originally proposed at the 1987 meeting of the Society for Computers in Psychology as a response to the needs of psychologists for information about psychology-related software and its users (Anderson, Hornby, \& Bozak, 1988). COMPsych was subsequently established in 1988 as a clearinghouse for software information related to psychology instruction, research, and practice. COMPsych provides information about software and users related to all hardware platforms and encourages the sharing of information among its users. Since its implementation, COMPsych has attempted to respond to the developing needs of psychologists and to new advancements in computer technology by expanding its database and enhancing its services. COMPsych, originally accessible via modem, electronic mail, and physical mail (Anderson \& Hornby, 1989), is now also accessible via anonymous ftp and gopher. The COMPsych Software Catalog is also published periodically in hard-copy form in the journal Computers in Human Services. Most recently, COMPsych has been designated as the official archive and distribution site for all PC-based software and normative data files described in the journal Behavior Research Methods, Instruments, \& Computers. To some extent, this new COMPsych service parallels a service provided by MacPsych (Huff \& Sobilof, 1993) for Macintosh-based software and files.

Correspondence concerning this article should be addressed to $P$. A. Hornby, Department of Psychology, State University College, Plattsburgh, NY 12901 (e-mail: hornbypa@snyplava.bitnet).

\section{Joining COMPsych}

A comprehensive description of the COMPsych service, methods of access, and a summary of early activity on the system has been presented by Anderson and Hornby (1989). At the present time, COMPsych has over 600 registered users, and over 700 software titles are listed in its catalog. Individuals interested in obtaining further information about the service as well as a registration form should send a plain language request to compsych@ snyplava.bitnet or compsych@splava.cc.plattsburgh.edu. Those without access to electronic mail may contact COMPsych by modem at (518) 564-3372 $(2400, N, 8,1)$ or by writing to COMPsych, Department of Psychology, SUNY, Plattsburgh, NY 12901. Individuals who complete the COMPsych registration form will be included in the directory of users and those providing electronic mail addresses will receive the COMPsych Newsletter. The Newsletter contains announcements of general interest, a list of all new software titles added to the COMPsych catalog since the last newsletter, and a listing of programs and data files added to the archive.

\section{Files Available From COMPsych}

The files in the COMPsych system are stored at the State University of New York College at Plattsburgh. These are available in a publicly accessible directory. Files stored in the COMPsych system are of two types. General COMPsych files consist of BULLETINS containing information about psychology software, software users, conferences, publications, and other announcements of interest to psychology software users. These files are 
stored in ASCII format and have the extension .txt. An index to these files is available as 00index.txt. In addition, files in the software archive consist of software and normative data files that have been previously described in the journal Behavior Research Methods, Instruments, $\&$ Computers. An index to the software archive is available as 00 index.sft. These files are stored in compressed form and have the extension .zip. Files that have the .zip extension must be uncompressed by using the software PKUNZIP (R). Contact your computing support center for information regarding the availability and use of this software.

\section{Getting Files From COMPsych by Using ftp}

All COMPsych files are available using anonymous file transfer protocol (ftp). Anonymous $\mathrm{ftp}$ is a form of $\mathrm{ftp}$ that does not require you to have an account on the machine that you are attempting to log on to. The site where COMPsych resides is gluon.hawk.plattsburgh.edu. General COMPsych files are located in the directory pub/compsych, and the software archive is located in pub/compsych/brmic. A description of the current file structure as well as any important notices regarding the system will be posted in a file called OOREADME.FIRST in the pub/ compsych directory. Your local computer support center should be able to provide guidelines for using ftp to obtain files from remote sites such as COMPsych. In general, the process for getting files from COMPsych to your PC consists of seven steps: (1) logging on to a machine that can connect to COMPsych using ftp, (2) connecting to COMPsych using ftp, (3) moving to the directory containing the files that you want to transfer, (4) setting the default mode for transferring the appropriate files (ASCII for .txt files and image for .zip files), (5) transferring the files you want to the machine you are logged on to, (6) downloading the files from the machine to your PC, and (7) for compressed files, uncompressing the files using pkunzip. When logging on to COMPsych, use the name "anonymous" and your user id as your password. The Appendix presents a sample ftp session with COMPsych. The italicized sections [within square brackets] provide an explanation of each step. If you experience difficulty in connecting to COMPsych by ftp or in transferring files, please request help from your local computing support center. If the difficulty is identified as a problem with our system, please send a message by electronic mail to compsych@snyplava.bitnet or compsych@ splava.cc.plattsburgh.edu with a description of the problem.

\section{Obtaining Files by Using Gopher}

In order to access COMPsych by gopher you must have Internet gopher client software on your local system. Gopher is a client/server application. The user interacts with a gopher client which provides the interface. The primary purpose of gopher is to allow the user to navigate the Internet through a series of menus. Once you are connected to the remote site via gopher, transfer of files is a simple process of selecting the desired files from the list presented. The file selected will be transferred to your site and you will be given the option to save it on your machine. All general COMPsych files, index files, and software files are available on the SUNY Plattsburgh Gopher Server. Depending on the features of your gopher client, it may not be possible for you to obtain program (binary) files by using gopher. If your gopher client does not support the transfer of binary files, follow the procedures outlined above for obtaining files by using ftp. Your local computer support center should be able to provide you with additional information about using your gopher server to access COMPsych.

\section{Submitting Files to the Archive}

Two types of files are appropriate for submission to COMPsych. Information to be included in general COMPsych BULLETINS can be submitted as ASCII text files and sent to compsych@snyplava.bitnet or compsych@ splava.cc.plattsburgh.edu. These include user registrations, new software descriptions (from developers and publishers), software reviews (from users), conference announcements, new publications dealing with computers in psychology, job openings, and general announcements or requests for inclusion in the Newsletter. Specific forms are available for software descriptions, software reviews, and user registrations, and these will be provided upon request. These forms are also available by ftp as described above.

Software and normative data files for inclusion in the software archive must be based on articles published in Behavior Research Methods, Instruments, \& Computers. Authors of such articles wishing to have their files included in the archive must follow specific guidelines for submission. Guidelines will be provided by COMPsych upon the request of the author or by the journal editor. These guidelines detail the appropriate documentation that must accompany the submission as well as procedures for compressing, bundling, and shipping the resulting file(s). In addition, authors should include a statement in their published article to the effect that the software and/or files described in the article may be obtained from COMPsych.

\section{COMPsych Responsibilities}

We hope that the addition of ftp and gopher access to COMPsych, as well as the establishment of the software and data file archive, will enhance the flexibility and overall value of the service. As a noncommercial enterprise, COMPsych is largely dependent on its users for input and assumes no responsibility for the quality or accuracy of the materials distributed. Any product described in the catalog should be fully investigated before a decision about acquisition is made. Also, COMPsych assumes no responsibility for providing support for installing or using any software or data files obtained from the archive. Authors of these products will include in their descriptions a statement about the level and availability of support for their products. In addition, COMPsych assumes no responsibility for any negative consequences of using software or 
information obtained from the archive. Finally, comments and suggestions regarding the COMPsych system are always welcome and should be submitted by electronic mail or physical mail to the addresses provided above.

\section{REFERENCES}

ANDERSON, M., \& Hornby, P. (1989). COMPsych: A description and progress report. Behavior Research Methods, Instruments, \& Computers, 21, 205-208.

ANDERSON, M., Hornby, P., \& BozaK, D. (1988). COMPsych: A computerized software information system. Behavior Research Methods, Instruments, \& Computers, 20, 243-245.

HuFf, C. \& SobILofF, B. (1993). MacPsych: An electronic discussion list and archive for psychology concerning the Macintosh computer. Behavior Research Methods, Instruments, \& Computers, 25, 60-64.

\section{APPENDIX \\ An Annotated ftp Session}

1. Log in to your local Internet machine.

2. At your system prompt type "ftp gluon.hawk.plattsburgh. edu" and hit return. After a brief delay you should see

Username [your user ID]:

[This indicates that a connection has been established between your Internet machine and the computer at SUNY Plattsburgh that is the COMPsych ftp site. If you do not get this prompt, it means that either there is something wrong with the Internet or there is a problem at one of the sites. Quit ftp by typing "bye" and contact your local computer support center.]

3. Type "anonymous" and hit return. You should see Password:
4. Type your complete user code, user name, and node address, and hit return. You should then see

$$
\text { FTP }>
$$

[This indicates that you have successfully logged on to the machine at Plattsburgh via ftp.]

5. Type "cd pub" and hit return, and then type "cd compsych" and hit return.

[This will get you to the directory where the general COMPsych files are stored. When you are in the COMPsych directory, use "cd brmic" to move to the software archive. To return up one level (to the COMPsych directory), simply enter "cd" and hit return.]

6. Type "ls" and hit return to list the files that are available in the directory.

7. Type "image" or "ASCII" and hit return to set the default transfer mode for the type of file you want to transfer.

["Image" should be used to transfer any file with the .zip extension. "ASCII" should be used to transfer files with the .txt extension. Once changed, the default transfer mode will remain the same until you change it again.]

8. Type "get" followed by the filename and extension that you wish to receive, and hit return.

[This will transfer one file. Continue to transfer files of the same type using the "get" command for each file.]

9. Type "bye" and hit return to log out from the COMPsych machine and return to your local system. 